\title{
Approches de mesure du concept de performance : Une revue de la littérature
}

\section{Approaches to measuring the concept of performance: A review of the literature}

\author{
HASSAS Mohammed (Doctorant) \\ Département des sciences économiques, \\ Faculté des Sciences Juridiques, Economiques et Sociales, \\ Université Mohamed Premier - Oujda, Maroc \\ JABBA Nozha (Doctorante) \\ Département des sciences économiques \\ Faculté des Sciences Juridiques, Economiques et Sociales, \\ Université Mohamed Premier - Oujda, Maroc \\ BENTAHAR Abdelrhani (Professeur) \\ Département des sciences économiques \\ Faculté des Sciences Juridiques, Economiques et Sociales \\ Université Mohamed Premier - Oujda, Maroc
}

\begin{abstract}
Résumé : Le concept de performance en tant que notion fondamentale en sciences de gestion, a fait l'objet d'une littérature intense depuis plusieurs décennies. Tout un corpus théorique fondé sur la finance, le rendement des actifs, des passifs et des ratios de rentabilité, avait été élaboré pour évaluer la performance du secteur privé. Plus récemment, la notion de performance a suscité un regain d'intérêt en lien avec la mondialisation de la concurrence. L'objet de cet article est de faire une synthèse de la littérature sur les différentes approches de mesure de la performance. Le but est de contribuer à rendre intelligible les facteurs de compréhension de ce qui détermine qu'une organisation à but non lucratif est efficace et performante.
\end{abstract}

Mots-clés : Performance; Efficacité; concurrence; finance.

Abstract: The concept of performance as a fundamental concept in management sciences has been the subject of intense literature for several decades. A whole theoretical corpus based on finance, the return on assets, liabilities and profitability ratios had been developed to assess the performance of the private sector. More recently, the concept of performance has sparked renewed interest in connection with the globalization of competition. The purpose of this article is to summarize the literature on the different approaches to performance measurement. The aim is to help make intelligible the factors for understanding what determines whether a non-profit organization is effective and efficient.

Keywords: Performance; Efficiency; Competition; finance. 


\section{Introduction}

Le terme performance est l'un de ces mots qui sont très utilisés, notamment en gestion. On le trouve dans différentes disciplines : en finance, en contrôle de gestion, en marketing, en gestion des ressources humaines, etc. En outre, le concept de la performance occupe une place importante dans le domaine de la recherche en sciences de gestion. Plusieurs auteurs se sont intéressés à la mesure des facteurs expliquant la performance des organisations.

Le concept de performance "définit l'accomplissement d'une action... » selon Michel Stella Ravelomanantsoa (2009) p.23)(1). D'ailleurs, ce même auteur (op.cit)(1) distingue trois approches différentes de la performance.

- L'approche littéraire où la performance est conçue comme « le résultat obtenu d'une tâche ou le chiffre qui mesure ce résultat».

- L'approche taylorienne où la performance devient le « synonyme de productivité des moyens mis en œuvre ». Le concept de performance est également rapprochée aux notions d'efficacité et d'efficience (Neely et al., 1995) (2).

- L'approche étymologique où la performance prend le sens d'action ou de résultat positif. De même qu'il est souvent associé aux notions de succès ou d'exploit. Ainsi, on parle de succès d'une action, de l'exploit d'une athlète ou de la performance d'une machine. De même qu'il traduit le succès dans l'amélioration de la qualité des produits et des services ou le degré de satisfaction des besoins de la clientèle en termes de qualité et de délais.

L'efficacité organisationnelle est l'un des éléments fondamentaux de la théorie des organisations (Baruh \& Ramalho, 20) (3) et (Goodmann \& Pennings, 1980) (4). Goodman et Pennings (1977)(5) soutiennent que l'efficacité est au cœur de l'étude de l'analyse organisationnelle et qu'une théorie des organisations devrait inclure l'étude de la construction de l'efficacité. Le problème est aussi ancien que la recherche organisationnelle elle-même (Cameron, 1980) (6), (Kalliath, \& Bluedorn et Gillespie, 1999) (7), (Shilbury \& Moore, 2006) (8).

Les études du concept de performance, vont s'intensifier entre les années 60 et les années 80 notamment celles de (Yuchtman \& Seashore 1967) (9), de (Cameron \& Whetten 1983) (10) et de (Quinn \& Rohrbaugh 1981, 1983) (11). Parmi les auteurs qui se sont chargés de répertorier ces théories les méthodes et approches de descriptions de l'efficacité organisationnelle, il y a Forbes (1998) (12) et Rojas (2000)(13).

La découverte de caractéristiques distinctives entre des organisations efficaces et inefficaces est le principal défi de l'évaluation organisationnelle. Différentes approches de l'efficacité ont été avancées par les théoriciens. Ainsi, Seashore et Yuchtman pour qui l'efficacité organisationnelle est assimilée à la capacité d'une organisation à exploiter l'environnement dans l'acquisition de ressources critiques (Seashore \& Yuchtman , 1967) (14).

Pour les dirigeants et gestionnaires, l'efficacité organisationnelle (OE) est en effet, un concept important pour comprendre si leurs organisations sont efficaces pour atteindre les résultats escomptés et réaliser leurs objectifs. Pour eux, l'amélioration de l'efficacité globale de l'organisation est un objectif clé pour qu'elle puisse rivaliser et survivre dans un environnement en mutation.

Malgré un intérêt professionnel et académique évident pour le sujet, il subsiste encore confusions et controverses sur ce qui constitue l'efficacité organisationnelle et comment la mesurer. L'absence de consensus sur une définition universelle accentue ce problème. Au fait, on est obligé de constater avec Forbes (1998) (12) et Shilbury\& Moore(2006) (8) que l'efficacité organisationnelle signifie différentes choses pour des personnes différentes. 
A travers une longue histoire, les théoriciens ont développé divers modèles et approches de l'efficacité organisationnelle reflétant différentes valeurs et sensibilités théoriques concernant l'efficacité. Walton \& Dawson, (2001) (15) et Herman \& Renz (1997) (16) ont déclaré qu'il existe autant de modèles d'efficacité qu'il y a de modèles d'organisations.

Pour cela et à travers une revue de la littérature, essentiellement anglophone, nous allons essayer de présenter différentes approches et différents niveaux d'analyse pour mesurer l'efficacité organisationnelle. On distingue au moins quatre grandes approches :

- les modèles d'objectif (Etzioni, 1960) (17), Price (1972)(18); et Scott (1977) (19),

- le modèle du système de ressource (Yuchtman\&Seashore,( 1967) (9),

- l'approche de processus interne (Pfeffer, 1977) (20), (Steers, 1977(21)),

- le modèle des circonscriptions multiples (Connolly, \& Deutsch, 1980) (22); Tsui, 1990; (23) Zammuto, 1984) (24) et la CVA (Quinn \&Rohrbaugh, 1981, 1983) (11).

\section{Le modèle d'objectif}

Le modèle d'objectif est le plus connu par ce que le plus largement appliqué. Plusieurs formulations ont été présentées. Mais c'est celle d'Etzioni(1960)(17) qui est souvent retenue. Elle stipule que «plus l'organisation se rapproche de l'atteinte de ses objectifs, plus elle est efficace » (Cameron, 1980, p.67)(6).

Aussi claire soit-elle, cette approche a ses propres limites comme l'ont souligné (Cameron, 1980(6); (Scott, 1987)(26); et (kahn, 1977)(25). C'est un modèle qui suppose que les objectifs soient clairement identifiables, suffisamment stables et mesurables dans le temps. Autrement dit, l'organisation doit avoir une image claire de ce qu'elle veut réaliser dans des délais déterminés et moyennant un budget bien défini afin que les résultats puissent refléter réellement les objectifs de l'organisation sans confusion aucune avec ceux reflétant les intérêts individuels ou de groupes spécifiques au sein de cette même organisation. Or, réunir simultanément de telles conditions est un pari difficilement tenable (Cameron, 1980)(6); (Herman \& Renz, 1999)(27). En effet, les objectifs sont enclins de changer au gré des circonstances politiques et économiques. De même que l'ambiguïté qui pourrait entacher leur formulation pourrait conduire à des interprétations complétement différentes ainsi qu'à des problèmes de mesure. C'est pourquoi, de nombreux chercheurs se sont orientés vers des approches moins centrées sur les aspects spécifiquement économiques.

\section{Le modèle du système de ressources}

La deuxième approche met l'accent sur l'acquisition des ressources nécessaires à une organisation. A l'origine, elle a été formulée par Seashore \&Yuchtman dans deux écrits réalisés tous les deux en 1967(Seashore \&Yuchtman, 1967(14); Yuchtman \&Seashore, 1967(9)) avant de connaitre de nouvelles versions légèrement aménagées (Georgopolous \&Tannenbaum, 1957(28)et (Steers, 1975(29)). C'est une approche qui est supposé remédier aux limites de l'approche d'objectifs. L'efficacité y est définie comme la capacité d'une organisation à exploiter son environnement dans l'acquisition de ressources rares et précieuses pour sa survie. On dira qu'une organisation est efficace lorsqu'elle est en mesure de pouvoir acquérir les ressources vitales de son environnement externe. 
Par rapport à l'approche d'objectifs, cette deuxième approche est plus globale dans la mesure où l'atteinte des objectifs est possible à partir de l'instant où l'organisation réalise un avantage concurrentiel (ce qui prouve son efficacité) par rapport aux organisations concurrentes à travers l'acquisition d'intrants (ressources) essentiels pour sa survie à long terme.

A l'instar de l'approche d'objectifs, l'approche de système de ressources a fait l'objet de nombreuses critiques parmi lesquelles on peut citer au moins deux :

- La première critique adressée à cette approche réside dans le fait qu'elle se concentre sur les moyens nécessaires pour atteindre l'efficacité plutôt que sur l'efficacité de l'organisation ellemême (Love, P.D. et Skitmore, M.R.1996, p.6)(30). Sans nier l'importance des ressources dans la survie d'une organisation, les deux auteurs semblent suggérer de ne pas négliger d'autres aspects qui affectent la performance de l'organisation.

- La deuxième a été formulée par Cameron (1984)(31) pour qui cette approche serait idéale s'il était possible de mettre au claire la relation de cause à effet entre ressources et performance. Autrement dit, cette relation pourrait donner lieu à des interprétations remettant en cause même partiellement les conclusions de Seashore \&Yuchtman.

\section{Le modèle des processus organisationnels internes}

La troisième approche d'efficacité est dénommée modèle des processus organisationnels (Pfeffer, 1977\& 20 Steers, 1977(21). Par rapport aux autres modèles qui mettent l'accent essentiellement sur les déterminants pouvant être qualifiés d'externes, celui-ci est focalisé sur les composantes internes du processus organisationnel comme déterminant de la performance de l'organisation. Par composantes internes, il est fait allusion à tout ce qui est en rapport avec le fonctionnement interne de l'organisation (Cameron, 1980)(6)et (Shilbury \& Moore, 2006)(8), comme par exemple les choix effectués, les décisions prises, les informations existantes, leur mode de circulation, les systèmes de gestion, la confiance qui règne au sein de l'organisation etc....

Les processus par contre reposent sur toutes les procédures permettant une meilleure articulation de ces composantes internes devant conduire à l'efficacité de l'organisation (Pfeffer, 1977)(20). On peut dire alors que le modèle des processus internes fonctionne lorsque les processus et procédures internes conduisent à des résultats probants (Cameron, 1980)(6).

\section{Le modèle de circonscriptions multiples}

Le quatrième modèle est l'approche (stratégique) à circonscriptions multiples (Connolly et al., 1980)(22). C'est un modèle qui a suscité un intérêt croissant au cours des années 1970, ce qui a favorisé le développement de nombreuses versions de ce modèle (D'Aunno, 1992)(32)); (Kanter\& Brinkerhoff,1981)(33) et(Tsui, 1990)(23); Zammuto,1984)(24). Connolly et al. (1980)(22) ont fait valoir que les modèles précédents - l'approche par objectifs et les approches par différents systèmes sont inadéquats car ils n'utilisent qu'un seul ensemble de critères d'évaluation. C'est pourquoi, il faut trouver une autre approche plus complète. C'est l'idée du modèle des circonscriptions multiples.

L'idée de base consiste à dire que chaque organisation a ses propres constituants ou parties prenantes et que chaque constituant définie ses propres critères d'évaluation de l'efficacité de l'organisation. Selon cette approche, l'organisation est efficace à partir de l'instant où les constituants stratégiques ont 
une satisfaction minimale (Cameron, 1980, p.67)(6). Cela signifie que même si une organisation a besoin pour sa survie de l'ensemble des parties prenantes internes (staff et bénéficiaires) et externes (bailleurs de fonds et communautés), son efficacité dépend essentiellement de la satisfaction des demandes des seules groupes de partenaires considérés comme stratégiques dans le sens où de par leur importance peuvent menacer la survie de l'organisation (Robbins, 1990)(34). Il est fait ici allusion essentiellement aux parties prenantes externes comme les bailleurs de fonds et les communautés.

\section{Conclusion}

En conclusion, il faut reconnaitre qu'à présent, les différents modèles d'efficacité organisationnelle présentés ont certes leurs avantages théoriques. Cependant, comme on l'a vu, chacun d'eux a ses propres limites. Nous déduisons, que tant qu'il n'y a pas de consensus général sur la définition de l'efficacité organisationnelle, il est difficile de compter sur un modèle plutôt que sur un autre. Cameron (1981)(35) a soutenu qu'une vision unilatérale ignore la complexité de l'efficacité organisationnelle et que les modèles d'efficacité devraient saisir de multiples dimensions. Une solution possible consisterait alors à élaborer un modèle de mesure complet qui intègre tous ces aspects évoqués par ces modèles. Certains auteurs estiment qu'il existe désormais un large consensus sur le fait que l'efficacité organisationnelle nécessite une approche multidimensionnelle (Chelladurai, 1987)(36); Forbes,1998(12); Herman, 1990(37); Herman \&Renz, 1999(16); Kalliath et al.,1999(7); Shilbury \& Moore, 2006(8); Sowa et al. , 2004)(38)).

\section{REFERENCES}

[1] Michel Stella Ravelomanantsao (2009), Contribution à la définition d'un cadre générique pour la définition, l'implantation et l'exploitation de la performance : application à la méthode ECOGRAI, thèse Univ de bordeaux, p.23).

[2] Neely, A., Adams, C., and Crowe, P. (2001) : Performance prism in practice. Measuring Business Excellence, 5(2), pp. 612. in Renaldi Safriansyah M(2010)

[3] Baruh, Y., \&Ramalho, N. 2006. Communalities and Distinctions in the Measurement of Organizational Performance and Effectiveness Across For-Profit and Nonprofit Sectors. Nonprofit and VoluntarySectorQuarterly, 35: 39-65. In ANNELINE BALDUCK et MARC BUELENS

[4] Goodmann, P. S., \& Pennings, J. M. 1980. Critical issues in assessing organizational effectiveness. In E.E. Lawler III, D.A. Nadler\& C. Camman (Eds.), Organizational Assessment. New York: Wiley. In ANNE-LINE BALDUCK et MARC BUELENS

[5] Goodmann, P. S., Pennings, J. M., \& Associates. 1977. New perspectives on organizational effectiveness. San Francisco: Jossey-Bass. In ANNE-LINE BALDUCK et MARC BUELENS

[6] Cameron, K.S 1980. Critical questions in assessing organizational effectiveness. Organizational dynamics, 4(2): 66-80.in Renaldi Safriansyah

[7] Kalliath, J., Bluedorn, A. C., \& Gillespie, D. F. 1999. A confirmatory factor analysis of the competing values instrument. Educational and Psychological Measurement, 59: 143-158. In ANNE-LINE BALDUCK et MARC BUELENS

[8] Shilbury, D., \& Moore, K. A. 2006. A study of organizational effectiveness for National Olympic Sporting Organizations. Non profit and Voluntary Sector Quarterly, 35: 5-38. In ANNE-LINE BALDUCK et MARC BUELENS

[9] Yutchman, E. \&Seashore, S. (1967). A system resource approach to organisational effectiveness, American Sociological Review 32, 891-903. In Renaldi Safriansyah

[10] Cameron, K. and D. Whetten (1983). "A model for teaching management skills." Journal of Management Education 8(2): 21 ; In Sharon G. Eng 
[10] Quinn, R. E., \&Rohrbaugh, J. 1981. A competing values approach to organizational effectiveness. Public Productivity Review, 5: 122-140. In ANNE-LINE BALDUCK et MARC BUELENS

[12] Forbes, D. P. (1998) 'Measuring the unmeasurable: empiricalstudies of non profit organization effectiveness from 1977 to 1997', Non profit and Voluntary Sector Quarterly 27: 183-202. In Sharon G. Eng

[13] Rojas, R. R. 2000. A review of models for measuring organizational effectiveness among for-profit and nonprofit organizations. Nonprofit Management \& Leaderschip, 11: 97-104.

[14] Seashore, S.E., Yutchman, E., (1967). Factor Analysis of Organisational Performance, Administrative Science Quarterly 12, 377-95. In Renaldi Safriansyah

[15] Walton, E. J., \& Dawson, S. 2001. Managers' perceptions of criteria of organizational effectiveness. Journal of Management Studies. Journal of Management Studies, 38: 173-199. In ANNE-LINE BALDUCK et MARC BUELENS

[16] Herman, R. D., and Renz, D. O. (1997) 'Multiple constituencies and the social construction of non profit organization effectiveness', Non profit and Voluntary Sector Quarterly 26(2): 185-206. In ANNE-LINE BALDUCK et MARC BUELENS

[17] Etzioni, A. 1960. Two approaches to organizational analysis: A critique and a suggestion. inANNE-LINE BALDUCK et MARC BUELENS

[18] Price, J. L. 1972. The study of organizational effectiveness. The Sociological Quarterly, 13:3-15. In ANNE-LINE BALDUCK et MARC BUELENS

[19] Scott, Richard W and John Meyer(1977), The Organizations of Societal Sectors: Propositions and Early Evidence, in The New Institutionalism in Organizational Analysis, pp. 108-140 in.FahimYoussofzai et Taïeb Hafsi, (2004)

[20] Pfeffer, J. 1977. Usefulness of the concept. In P. S. Goodmann \& J. M. Pennings (Eds.), New perspectives on organizational effectiveness: 132-143. San Francisco: Jossey-Bass. In ANNE-LINE BALDUCK et MARC BUELENS

[21] Steers, R. M. 1977. Organizational effectiveness: A behavioral view.Santa Monica: Goodyear. In ANNE-LINE BALDUCK et MARC BUELENS

[22] Connolly, T., Conlon, E., and Deutsch, S. (1980). Organizational effectiveness: A multiple constituencyapproach. Academy of Management Review, 5(2), pp. 211-217. In ANNE-LINE BALDUCK et MARC BUELENS

[23] Tsui, A. S. 1990. A multiple constituency model of effectiveness: An empirical examination at the Human Resource subunit level. Administrative Science Quarterly, 35: 458-483. In ANNE-LINE BALDUCK et MARC BUELENS

[24] Zammuto, R. F. 1984. A comparison of multiple constituency models of organizational effectiveness. Academy of Management Review, 9: 606-616. In ANNE-LINE BALDUCK et MARC BUELENS

[25] Kahn, R.L. (1977). Organisational effectiveness: an overview, In Renaldi Safriansyah

[26] Scott, Richard W., The Adolescence of Institutional Theory, Administrative Science Quarterly, 32, 1987, pp. $493-511$. in.FahimYoussofzai et Taïeb Hafsi, (2004)

[27] Herman, R. D., \& Renz, D. O. 1999. Theses on non profit organizational effectiveness. Non profit and Voluntary Sector Quarterly, 28: 107-126. In ANNE-LINE BALDUCK et MARC BUELENS

[28] GEORGOPOUlOS, Basil ; TANNENBAUM, Arnold, " Study of Organizational Effectiveness ». American Sociological Review, n²2, 1957, pp. 534-540. In Nathalie Audigier

[29] STEERS, Richard, « Problems in the Measurement of Organizational Effectiveness ». Administrative Science Quarterly, $\mathrm{n}^{\circ} 20,1975$, pp. 546-548. in Nathalie Audigier

[30] Love, Peter E.D. \&Skitmore, M. R. (1996). Approaches to Organisational Effectiveness Eds. Proceedings 12th Annual Conference and Annual General Meeting, The Association of Researchers in Construction Management, Sheffield Hallam University. Accessed from: http://eprints.qut.edu.au/archive/00004524 in Renaldi Safriansyah

[31] Cameron, KS (1984) L'efficacité de l'inefficacité. Dans B.M Staw et LL Cummings (éd.), Research in Organizational Behavior, Vol. 6, 235-285 in Sharon G.Eng 
[32] D'Aunno, T. 1992. The effectiveness of human service organizations: A comparison of models. In Y. Hasenfeld (Ed.), Human services as complex organizations. Thousand Oaks California: Sage. In ANNE-LINE BALDUCK et MARC BUELENS

[33] Kanter, R. M., \& Brinkerhoff, D. 1981. Organizational performance: Recent developments in measurement In R.H. Turner \& J.F.JR. Short (Eds.), Annual Review of Sociology. Palo Alto California: Annual Reviews. In ANNE-LINE BALDUCK et MARC BUELENS

[34] Robbins, S.P.(1990). Organisational theory: structure, design and applications (3rd Ed.), Englewood Cliffs: Prentice Hall. In Renaldi Safriansyah

[35] Cameron, K. 1981. Domains of organizational effectiveness in Colleges and Universities.in ANNE-LINE BALDUCK et MARC BUELENS

[36] Chelladurai, P. 1987. Multidimensionality and multiple perspectives of organizational effectiveness. Journal of Sport Management, 1: 37-47.in ANNE-LINE BALDUCK et MARC BUELENS

[38] Herman, R. (1990). Methodological Issues in Studying the Effectiveness of Non governmental and Nonprofit Organizations. Nonprofit and Voluntary Sector Quarterly, 19 (3), 293-306. In Pat Bradshaw, Vic Murray, Jacob Wolpin

[38] Sowa, J., Selden, S., and Sandfort, J. (2004). No longer unmeasurable?: A multidimensional integrated model of non profit organizational effectiveness. Nonprofit and VoluntarySectorQuarterly, 33(4), pp. 711-728. In ANNE-LINE BALDUCK et MARC BUELENS

\section{BIBLIOGRAPHIE}

[1] ANNE-LINE BALDUCK et MARC BUELENS , 2008 , A TWO-LEVEL COMPETING VALUES FRAMEWORK TO MEASURING NONPROFIT ORGANIZATIONAL EFFECTIVENESS ; Vlerick Leuven Gent Working Paper Series 2008/19

[2] Fahim Youssofzai et Taïb Hafsi, (2004) ,Strategic management and performance of humanitarian systems ; Cahier de recherche $\mathrm{N}^{\circ}$ 06-23-03 Août 2004

[3] Michel Stella RAVELOMANANTSOA(2009), Contribution à la définition d'un cadre générique pour la définition, l'implantation et l'exploitation de la performance : application à la méthode ECOGRAI, thèse Univ de bordeaux.

[4] Nathalie Audigie , (2008), L'évaluation organisationnelle au sein de services non marchands : quelques éléments de réflexion, Presses universitaires de Bordeaux. 4.

[5] Pat Bradshaw, Vic Murray and Jacob Wolpin, (1992), Do Non profit Boards Make a Difference? An Exploration of the Relationships Among Board Structure, Process, and Effectiveness, in Nonprofit and Voluntary Sector Quarterly • September $1992 ; 21$;

[6] Renaldi Safriansyah M ( 2010) ; Measuring Organisational Effectiveness; From Theoretical Model to Practical Use. , Queensland University of Technology, Brisbane, Australia

[7] Rojas, R. R. 2000. A review of models for measuring organizational effectiveness among for-profit and non profit organizations. Nonprofit Management \& Leaderschip, 11: 97-104.

[8] Sharon G. Eng (2007), Defining NGO organisational effectiveness, 1st presentedat: International Society for Third Sector Research (ISTR)5th ISTR Asia and Pacific Regional Conference Manila, Philippines, October 17-18, in Indonesia: an unfolding journey 